\title{
Clinico-pathological study of ovarian tumors in Goa medical college: a tertiary care centre in Goa, India
}

\author{
Manjusha Jindal*, Dweep Jindal, Mrinalini Sahasrabhojane, Viraj Naik
}

Department of Obstetrics and Gynecology, Goa Medical College, Bambolim, Goa, India

Received: 13 August 2019

Accepted: 07 September 2019

*Correspondence:

Dr. Manjusha Jindal,

E-mail: manjushajindal@gmail.com

Copyright: (c) the author(s), publisher and licensee Medip Academy. This is an open-access article distributed under the terms of the Creative Commons Attribution Non-Commercial License, which permits unrestricted non-commercial use, distribution, and reproduction in any medium, provided the original work is properly cited.

\begin{abstract}
Background: Ovarian neoplasms are a distinct entity in women health care and are increasingly contributing to morbidity and mortality among women. The burden is not only related to the increasing incidence but also to the varied pathological features depending on the tissue of origin and pathogenesis. The study was carried out to find the prevalence and determine the clinical presentation and histo-pathological distribution of ovarian neoplasms. Management options were also noted.

Methods: It is a retrospective study carried out at Goa Medical College between January 2013 to December 2015 . All patients diagnosed and treated for ovarian neoplasm were included in the study. Data was tabulated using Microsoft Excel and descriptive statistical analysis was carried out using SPSS version 23.

Results: A total of 3111 patients were admitted in gynecology at Goa Medical College during the specified time period. Of these 358 cases were diagnosed with ovarian neoplasm. On histopathology 196 were benign tumors and 162 were reported to be malignant. Commonest presenting symptom was abdominal distention seen in $51.1 \%$ of the patients, pain in $44.4 \%$, followed by dyspepsia in $26.85 \%$. Epithelial tumors were most common (Benign - 39.3\%, Malignant $-41 \%$ ) followed by sex cord stromal tumors and germ cell tumors in $7.26 \%$ of cases.

Conclusions: Surface epithelial tumors were most common neoplasm. An alarming high no. of malignant tumors $(45.25 \%)$ was found in present study. $44.4 \%$ tumors presented in 41-50 years age group. Presenting complaints were vague and nonspecific leading to delay in diagnosis. Histological type correlates with prognosis; therefore, preponderance of histological type will guide treatment options and patient education with respect to epidemiology.
\end{abstract}

Keywords: Benign, Germ cell, Histopathological type, Malignant, Ovarian neoplasm, Surface epithelial

\section{INTRODUCTION}

Ovary, the Female gonad is very complex in its embryology and histology. Due to complex histology, the ovarian tumors have different cell origins, complex nature and varied clinical presentation. ${ }^{1}$ They present at any age but $90 \%$ of benign tumors are seen in childbearing age. ${ }^{2}$ Benign tumors are cystic in nature and presence of solid components increases suspicion of malignancy. The potential malignant behaviour of some ovarian tumors causes dilemma with respect to conservative/definitive surgery.

Malignant ovarian tumors comprise of $3.6 \%$ of all cancers in women. It is the seventh most common cancer among women (age standardised incidence rate being $6.1 / 100,000)$ and fifth leading cause of cancer deaths (4.3\%) globally with age standardised mortality rate being $3.8 / 100,00 .^{3}$ In developed countries, more than $90 \%$ of malignant ovarian tumors are epithelial in origin, 
$5 \%-6 \%$ of tumors constitute sex cord-stromal tumors, and $2 \%-3 \%$ are germ cell tumors. ${ }^{4}$ The common epithelial ovarian cancers (EOCs) include high-grade serous $(70 \%)$, endometrioid $(10 \%)$, clear cell $(10 \%)$, mucinous $(3 \%)$, and low-grade serous carcinomas $(<5 \%){ }^{4}$

Ovarian tumors are not detected early as they occupy available space in pelvis and abdomen for expansion. They seek attention after achieving big size. ${ }^{5}$ In malignant tumors, the symptoms are vague, and therefore patients unfortunately present in late stages. ${ }^{6}$

The clinical presentation and imaging findings provide diagnostic clue in some cases. The laterality of the tumor also indicates their nature, as sex cord stromal tumors are usually unilateral. ${ }^{2}$ The role of pathologist is important in establishing correct diagnosis and effective treatment. ${ }^{1}$

Study of clinical presentation, histopathological pattern, management options and response not only help for prognostication, but also redefines management. With government focus on maternal and child health services, these women are usually neglected. Health seeking behaviour is also guided by social and economic factors.

Present study is undertaken to find out the prevalence, age distribution and histological pattern prevalent in our population so as to guide treatment options and provide patient education.

\section{METHODS}

This is retrospective descriptive study carried out in the Department of Obstetrics and Gynecology, Goa Medical College, A tertiary care center of state of Goa from January 2013 to December 2015. Study subjects consisted of admitted patients in gynecology ward; diagnosed as case of ovarian tumor on basis of clinical and imaging findings and confirmed by laparotomy/ laparoscopy findings and histopathology. Pelvic masses other than ovarian tumor on histopathology were excluded from the study. The clinical data specially included was physical symptoms, examination findings. Study data included age at diagnosis, obstetric history, menstrual history, personal and family history, and presenting complaints. Imaging studies included USG in all cases and CECT/MRI wherever indicated. Laparotomy/laparoscopy findings and treatment was noted. Histopathology details were noted down and classification was done according to International Classification of diseases (WHO classification 2019). Data was tabulated using microsoft excel and expressed in terms of percentage and means with standard deviation.

Approval of Institutional Ethics committee of Goa Medial College was taken.

Objectives of this study were to study the proportion of ovarian tumors. To study the distribution of ovarian neoplasms as per WHO classification. To study their age distribution. To study the clinical presentation and correlation to histopathology.

\section{RESULTS}

There were 358 cases of ovarian tumors among 3311 Gynecological admissions during three years period from January 2013 to December 2015. Thus, the proportion of ovarian tumors among all gynecological admissions was $10.8 \%$. Among these, $196(54.75 \%)$ were benign tumors and162 $(45.25 \%)$ cases were malignant tumors including four cases of borderline malignancy.

\section{Histo-pathology}

The surface epithelial tumors were most common $(80.45 \%)$ as per WHO classification as shown in Table 1.

Table 1: Distribution of tumors on histo-pathological basis.

\begin{tabular}{|lllll|}
\hline Histology & Benign & Malignant & Total & Total \%age \\
\hline Surface epithelial & $141(39.4 \%)$ & $147(41 \%)$ & 288 & $80.45 \%$ \\
\hline Sex cord stromal & $34(9.5 \%)$ & $6(1.67 \%)$ & 40 & $11.17 \%$ \\
\hline Germ cell & $21(5.86 \%)$ & $5(1.4 \%)$ & 26 & $7.26 \%$ \\
\hline Secondary & - & 2 & 2 & $0.56 \%$ \\
\hline Undifferentiated & $\mathbf{1 9 6}$ & 2 & 2 & $0.56 \%$ \\
\hline Total & & $\mathbf{1 6 2}$ & $\mathbf{3 5 8}$ & $\mathbf{1 0 0 \%}$ \\
\hline
\end{tabular}

Table 2 shows the histopathological pattern of benign ovarian tumors. In benign tumors serous epithelial tumors were most common followed by mucinous tumors.
Table 3 shows that epithelial tumors comprised the bulk of malignant tumors $(90.73 \%)$. There were 6 cases of sex cord stromal tumors, 5 cases of germ cell tumors. And 2 cases of Kruckenberg tumor. 
Table 2: Distribution of benign ovarian tumours as per histo-pathology.

\begin{tabular}{|lll|}
\hline Histology & $\mathrm{N}=196$ & $\%$ \\
\hline Serous tumor & 95 & 48.47 \\
\hline Mucinous tumor & 38 & 19.38 \\
\hline Endometroid tumor & 3 & 01.53 \\
\hline Brenner tumor (transitional cell) & 3 & 01.53 \\
\hline Mixed & 2 & 01.02 \\
\hline Steroid cell & 2 & 01.02 \\
\hline Adeno-fibroma & 18 & 9.19 \\
\hline Fibroma thecoma & 14 & 7.14 \\
\hline Mature cystic teratoma & 21 & 10.72 \\
\hline
\end{tabular}

Table 3: Distribution of malignant ovarian tumours including borderline malignancy as per histo-pathology.

\begin{tabular}{|llll|}
\hline \multirow{4}{*}{$\begin{array}{l}\text { Epithelial } \\
\text { tumors }\end{array}$} & Serous tumor & $\mathrm{N}=162$ & $\%$ \\
\cline { 2 - 4 } & Mucinous tumor & 11 & 74.69 \\
\cline { 2 - 4 } & Endometroid tumor & 3 & 06.79 \\
\cline { 2 - 4 } & Clear cell & 12 & 01.85 \\
\hline $\begin{array}{l}\text { Sex cord } \\
\text { stromal } \\
\text { tumor }\end{array}$ & Mixed mullerian tumor & 1 & 0.6 \\
\cline { 2 - 4 } $\begin{array}{l}\text { Germ cell } \\
\text { tumors }\end{array}$ & Granulosa cell & 5 & 3.1 \\
\cline { 2 - 4 } & Immature teratoma & 2 & 1.85 \\
\hline Secondaries & Kruckenberg & 2 & 1.24 \\
\hline & Undifferentiated & 2 & 1.24 \\
\hline
\end{tabular}

Age

Surface epithelial benign tumors were seen at mean age of $42.06 \pm 5.8$ years while malignant tumors were found at mean age of $52.97 \pm 6.27$ years. Malignant germ cell tumors were seen at mean age of $23.5 \pm 8.3$ years and benign tumors at mean age of $37.3 \pm 5.3$ years. Table 4 shows mean age of cases with various ovarian tumors.

Table 4: Mean age of cases with ovarian tumors.

\begin{tabular}{|c|c|c|c|}
\hline \multicolumn{4}{|c|}{ Mean age in years } \\
\hline Histology & Benign & Borderline & Malignant \\
\hline Surface epithelia & $42.06 \pm 5.8$ & $45.46 \pm 1.2$ & $52.97 \pm 6.27$ \\
\hline Serous & $38.84 \pm 8.7$ & 43.7 & $53.8 \pm 7.4$ \\
\hline Mucinous & $41.31 \pm 6.4$ & $46.3 \pm 0.8$ & $56.4 \pm 5.6$ \\
\hline $\begin{array}{l}\text { Endometroid } \\
\text { tumor }\end{array}$ & $43.8 \pm 2.3$ & 46.4 & $57.1 \pm 3.2$ \\
\hline Clear cell & & & $44.3 \pm 8.9$ \\
\hline $\begin{array}{l}\text { Brenner tumor } \\
\text { (transitional cell), }\end{array}$ & $39.75 \pm 1.3$ & & \\
\hline Mixed & $46.6 \pm 0.8$ & & \\
\hline $\begin{array}{l}\text { Sex cord stromal } \\
\text { Tumor }\end{array}$ & $39.59 \pm 9.9$ & & $43 \pm 2.4$ \\
\hline Steroid cell & $24.8 \pm 1.9$ & & \\
\hline Adeno-fibroma & $42.8 \pm 2.7$ & & \\
\hline Fibroma thecomi & $46.66 \pm 3.4$ & & \\
\hline Granulosa cell & & & $43.1 \pm 2.6$ \\
\hline $\begin{array}{l}\text { Mixed mullerian } \\
\text { tumor }\end{array}$ & & & 43.6 \\
\hline Germ cell tumor & $37.3 \pm 5.3$ & & $23.5 \pm 8.3$ \\
\hline $\begin{array}{l}\text { Mature cystic } \\
\text { teratoma }\end{array}$ & $37.3 \pm 5.3$ & & \\
\hline Dysgerminoma & & & $26.1 \pm 9.2$ \\
\hline Immature terator & & & $20.9 \pm 1.3$ \\
\hline $\begin{array}{l}\text { Secondary } \\
\text { (Krucken berg) }\end{array}$ & & & $46.7 \pm 2.8$ \\
\hline Undifferentiated & & & $43.4 \pm 3.4$ \\
\hline
\end{tabular}

Table 5: Age wise distribution of ovarian tumors.

\begin{tabular}{|c|c|c|c|c|c|}
\hline $\begin{array}{l}\text { Age in } \\
\text { years }\end{array}$ & $\begin{array}{l}\text { Total no. of } \\
\text { tumors }\end{array}$ & $\begin{array}{l}\text { No. of Benign/ } \\
\text { borderline/malignant }\end{array}$ & Histopathology & $\begin{array}{l}\text { No. of Benign/ } \\
\text { borderline/malignant }\end{array}$ & $\%$ \\
\hline \multirow{5}{*}{$<20$} & \multirow{5}{*}{$4(1.1 \%)$} & \multirow{5}{*}{ 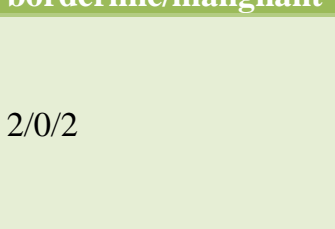 } & Surface epithelial & $2 / 0 / 0$ & 2 \\
\hline & & & Sex cord stromal & 0 & 0 \\
\hline & & & Germ cell & $0 / 0 / 2$ & 2 \\
\hline & & & Secondary & 0 & 0 \\
\hline & & & Undifferentiated & 0 & 0 \\
\hline \multirow{5}{*}{$21-30$} & \multirow{5}{*}{$28(7.8 \%)$} & \multirow{5}{*}{$25 / 0 / 3$} & Surface epithelial & $13 / 0 / 0$ & 13 \\
\hline & & & Sex cord stromal & $4 / 0 / 0$ & 4 \\
\hline & & & Germ cell & $8 / 0 / 3$ & $11(42.3 \%)$ \\
\hline & & & Secondary & 0 & 0 \\
\hline & & & Undifferentiated & 0 & 0 \\
\hline \multirow{5}{*}{$31-40$} & \multirow{5}{*}{$89(24.8 \%)$} & \multirow{5}{*}{$76 / 2 / 11$} & Surface epithelial & $53 / 2 / 8$ & $63(21.8 \%)$ \\
\hline & & & Sex cord stromal & $12 / 0 / 2$ & $14(35 \%)$ \\
\hline & & & Germ cell & $12 / 0 / 0$ & $12(46 \%)$ \\
\hline & & & Secondary & 0 & \\
\hline & & & Undifferentiated & $0 / 0 / 1$ & \\
\hline
\end{tabular}




\begin{tabular}{|c|c|c|c|c|c|}
\hline $\begin{array}{l}\text { Age in } \\
\text { years }\end{array}$ & $\begin{array}{l}\text { Total no. of } \\
\text { tumors }\end{array}$ & $\begin{array}{l}\text { No. of Benign/ } \\
\text { borderline/malignant }\end{array}$ & Histopathology & $\begin{array}{l}\text { No. of Benign/ } \\
\text { borderline/malignant }\end{array}$ & $\%$ \\
\hline \multirow{5}{*}{$41-50$} & \multirow{5}{*}{$159(44.4 \%)$} & \multirow{5}{*}{$88 / 2 / 69$} & Surface epithelial & $70 / 2 / 63$ & $135(46.9 \%)$ \\
\hline & & & Sex cord stromal & $17 / 0 / 4$ & $21(52.5 \%)$ \\
\hline & & & Germ cell & $1 / 0 / 0$ & \\
\hline & & & Secondary & $0 / 0 / 1$ & \\
\hline & & & Undifferentiated & $0 / 0 / 1$ & \\
\hline \multirow{5}{*}{$51-60$} & \multirow{5}{*}{$74(20.8 \%)$} & \multirow{5}{*}{$4 / 0 / 70$} & Surface epithelial & $3 / 0 / 68$ & $71(24.6 \%)$ \\
\hline & & & Sex cord stromal & $1 / 0 / 0$ & \\
\hline & & & Germ cell & 0 & \\
\hline & & & Secondary & $0 / 0 / 1$ & \\
\hline & & & Undifferentiated & 0 & \\
\hline \multirow{5}{*}{$>60$} & \multirow{5}{*}{$4(1.1 \%)$} & \multirow{5}{*}{$0 / 0 / 4$} & Surface epithelial & $0 / 0 / 4$ & \\
\hline & & & Sex cord stromal & 0 & \\
\hline & & & Germ cell & 0 & \\
\hline & & & Secondary & 0 & \\
\hline & & & Undifferentiated & 0 & \\
\hline Total & 358 & 19 & & & \\
\hline
\end{tabular}

Benign epithelial and sex cord stromal tumors were most common in 41-50 years while malignant epithelial tumors were seen in age group of 41-60 years. Germ cell tumors occurred in younger patients (21-40 years). The youngest patient was 16 years old, a case of endodermal sinus tumor while the 73 years old lady had serous cyst adenocarcinoma. Age wise distribution is shown in Table 5. Surface epithelial tumors were most common in all age groups. Benign surface epithelial tumors were more than malignant tumors till 40 years of age and after 40 years malignant tumors took preponderance. Most of the sex cord stromal tumors were seen in 31-50 years of age. Malignant germ cell tumors were seen before 30 years of age while mature cystic teratoma was seen between 21-40 years and only one case was at 43 years of age.

\section{Obstetric history}

The cases had no specific relation to parity as shown in Table 6.

Table 6: Para wise distribution of ovarian tumors.

\begin{tabular}{|lllll|}
\hline Parity & Benign & Borderline & Malignant & Total \\
\hline 0 & $56(28.6 \%)$ & & $17(10.6 \%)$ & $73(20.4 \%)$ \\
\hline 1 & $33(16.8 \%)$ & & $31(19.29 \%)$ & $64(17.9 \%)$ \\
\hline 2 & $51(26.1 \%)$ & $1(25 \%)$ & $44(28.17 \%)$ & $96(26.8 \%)$ \\
\hline 3 & $38(19.4 \%)$ & $2(50 \%)$ & $33(21.05 \%)$ & $73(20.4 \%)$ \\
\hline 4 & $16(8.1 \%)$ & $1(25 \%)$ & $18(11.48 \%)$ & $35(9.7 \%)$ \\
\hline$>4$ & $2(1 \%)$ & & $15(9.57 \%)$ & $17(4.8 \%)$ \\
\hline Total & $\mathbf{1 9 6}(\mathbf{1 0 0 \%})$ & $\mathbf{4 ( 1 0 0 \% )}$ & $\mathbf{1 5 8}(\mathbf{1 0 0 \%})$ & $\mathbf{3 5 8}(\mathbf{1 0 0 \%})$ \\
\hline
\end{tabular}

Table 7: Clinical presentation.

\begin{tabular}{|llll|}
\hline Symptom & Benign & Malignant & Total N=358\% \\
\hline Asymptomatic & $5(1.4 \%)$ & 0 & $5(1.4 \%)$ \\
\hline Infertility & $27(7.54 \%)$ & 0 & $27(7.54 \%)$ \\
\hline Pain abdomen & $102(28.5 \%)$ includes 3 cases of acute abdomen & $57(15.9 \%)$ & $159(44.4 \%)$ \\
\hline Abdominal distension & $92(25.7 \%)$ & $91(25.4 \%)$ & $183(51.1 \%)$ \\
\hline Amenorrhea & $3(0.83 \%)$ & 0 & $3(0.83 \%)$ \\
\hline Abnormal uterine bleeding & $16(4.47 \%)$ & $18(5.02 \%)$ & $34(9.5 \%)$ \\
\hline Dyspepsia & 0 & $96(26.8 \%)$ & $96(26.8 \%)$ \\
\hline Loss of weight & $2(0.56 \%)$ & $61(17.0 \%)$ & $63(17.6 \%)$ \\
\hline Urinary symptoms & $3(0.84 \%)$ & $6(1.68 \%)$ & $9(2.51 \%)$ \\
\hline Breathlessness & $6(1.58 \%)$ & $20(5.59 \%)$ & $26(7.26 \%)$ \\
\hline
\end{tabular}

* Few patients had multiple symptoms therefore the total no. exceeds actual cases. 


\section{Menstrual function}

$90(97 \%)$ women were regularly menstruating among benign ovarian tumors as compared to $99(61.1 \%)$ women having malignant tumor. Six $(3.06 \%)$ women were post-menopausal among benign tumors compared to $63(38.9 \%)$ women with malignant tumor.

\section{Clinical presentation}

The clinical presentation is as shown in Table 7. The predominant symptom in benign tumors was pain abdomen $(28.5 \%)$ followed by abdominal distension in $25.7 \%$ cases. Among malignant tumors dyspepsia was the predominant symptom in $26.8 \%$ followed by abdominal distension in $25.4 \%$ of all tumors. Among benign tumors serous cyst adenoma was commonest comprising of $48.47 \%$ followed by mucinous cyst adenoma (19.38\%) and mature cystic teratoma $(10.72 \%)$.

\section{Laterality}

Benign tumors were unilateral in 134 (68.36\%) cases while malignant tumors were bilateral in $94.9 \%$ cases and unilateral only in $5.1 \%$ cases (Table 8 ).

Table 8: Laterality of ovarian tumors.

\begin{tabular}{|lll|l|}
\hline Laterality & Benign & Borderline & Malignant \\
\hline Unilateral & $134(68.4 \%)$ & 1 & $8(5.1 \%)$ \\
\hline Bilateral & $62(31.6 \%)$ & 3 & $150(94.9 \%)$ \\
\hline Total & $\mathbf{1 9 6}$ & $\mathbf{4}$ & $\mathbf{1 5 8}$ \\
\hline
\end{tabular}

Table 9: Treatment options in benign tumors.

\begin{tabular}{|lll|}
\hline Type of Surgery & No. & $\%$ \\
\hline Cystectomy & 97 & 49.5 \\
\hline Ovariotomy & 28 & 14.3 \\
\hline Salpingo-ovariotomy & 12 & 6.2 \\
\hline Hysterectomy with ovariotomy & 59 & 30 \\
\hline Total & $\mathbf{1 9 6}$ & $\mathbf{1 0 0}$ \\
\hline
\end{tabular}

Table 10: Treatment options in malignant tumors.

\begin{tabular}{|lll|}
\hline Type of treatment & No. & $\%$ \\
\hline Ovariotomy & 4 & 2.5 \\
\hline $\begin{array}{l}\text { TAH with BSO with / without } \\
\text { omentectomy }\end{array}$ & 10 & 6.2 \\
\hline Surgery + Chemotherapy & 43 & 26.5 \\
\hline NACT+surgery +chemo & 61 & 37.7 \\
\hline NACT Awaiting surgery & 26 & 16.0 \\
\hline Palliative Chemotherapy & 18 & 11.1 \\
\hline Total & $\mathbf{1 6 2}$ & \\
\hline
\end{tabular}

\section{Management}

The treatment options were noted in both benogn and malignant ovarian tumors. The treatment was conservative in most of benign tumors as shown in Table 9.

As malignant tumors are diagnosed late, 87 cases $(53.7 \%)$ cases received neo-adjuvant chemotherapy (NACT). Table 10 shows the treatment options in malignant ovarian tumors.

\section{DISCUSSION}

The proportion of ovarian tumors among gynaecological admissions in our study was $10.85 \%$ while Yogambal et al, reported $5.4 \%(402 / 7492)$ cases to be of ovarian tumors among hospital admissions. ${ }^{2}$ The benign tumors comprised of $54.75 \%$, border line tumors were $1.12 \%$ and malignant tumors were $44.13 \%$. All the studies (Table 11) reported a high proportion of benign tumors ranging from 64-90\% while in our study benign tumors were $54.75 \%$. We had a large number of malignant tumors as ours is the only tertiary hospital catering to whole of population of Goa and neighboring areas from states of Maharashtra and Karnataka.

Table 11: Incidence of benign and malignant tumors in various studies.

\begin{tabular}{|c|c|c|c|}
\hline Study & Benign & Borderline & Malignant \\
\hline Yogambal et $\mathrm{al}^{2}$ & $78.6 \%$ & $0.75 \%$ & $20.65 \%$ \\
\hline Manoja et al $^{1}$ & $90 \%$ & - & $10 \%$ \\
\hline Shanthi $\mathrm{V}^{7}$ & $91.67 \%$ & - & $8.33 \%$ \\
\hline Ameena $^{8}$ & $64.57 \%$ & - & $35.43 \%$ \\
\hline Amod $S^{9}$ & $75.7 \%$ & $6.1 \%$ & $18.2 \%$ \\
\hline Swamy $\mathrm{G}^{10}$ & $71.6 \%$ & $3 \%$ & $25.4 \%$ \\
\hline Sarangan $\mathrm{A}^{11}$ & $89 \%$ & $4 \%$ & $7 \%$ \\
\hline Sumaira $Y^{12}$ & $89.71 \%$ & - & $10.29 \%$ \\
\hline Present study & $54.75 \%$ & $1.12 \%$ & $44.13 \%$ \\
\hline
\end{tabular}

The clinical presentation of the tumors was variable. Abdominal distension/mass was the commonest symptom $(51.1 \%)$ among all tumor followed by pain in $44.4 \%$ cases. Manoja et al, also reported mass per abdomen to be the commonest symptom in $40.8 \%$ followed by pain abdomen in $37.5 \%$ cases. In their study menstrual abnormality was seen in $10 \%$, GI disturbance in $6.7 \%$ and infertility in $2.5 \%$ cases. In the study by Sumaira et al and Yogambal et al, pain abdomen was the commonest symptom in $70.59 \%$ and $66.92 \%$ respectively followed by mass abdomen in $14.71 \%$ and $28.11 \%$ respectively as shown in Table 12.,12

Table 12: Clinical presentation of ovarian tumors in various studies.

\begin{tabular}{|lll|}
\hline Study & Mass abdomen & Pain abdomen \\
\hline Manoja et al $^{1}$ & $40.8 \%$ & $37.5 \%$ \\
\hline Sumaira et $\mathrm{al}^{12}$ & $14.71 \%$ & $70.59 \%$ \\
\hline${\text { Yogambal et } \mathrm{al}^{2}}^{2}$ & $28.11 \%$ & $66.92 \%$ \\
\hline Present study & $51.1 \%$ & $44.4 \%$ \\
\hline
\end{tabular}


Table 13: Clinical presentation in benign tumors in various studies.

\begin{tabular}{|c|c|c|c|c|c|c|c|c|}
\hline & Asymptomatic & Infertility & $\begin{array}{l}\text { Menstrual } \\
\text { abnormality }\end{array}$ & Pain & $\begin{array}{l}\text { Mass } \\
\text { abdomen }\end{array}$ & $\begin{array}{l}\text { Loss of } \\
\text { weight }\end{array}$ & GI symptoms & Misc. \\
\hline Manoja et $\mathrm{al}^{1}$ & - & $1.8 \%$ & $9.3 \%$ & $38.9 \%$ & $42.6 \%$ & - & $7.4 \%$ & - \\
\hline Shanti $\mathrm{V}$ et al ${ }^{7}$ & - & - & $13.99 \%$ & $36.36 \%$ & $39.16 \%$ & - & $10.49 \%$ & - \\
\hline $\begin{array}{l}\text { Present study } \\
(\mathrm{N}=196)\end{array}$ & $2.55 \%$ & $13.8 \%$ & $9.7 \%$ & $52 \%$ & $46.9 \%$ & $1.0 \%$ & - & $4.6 \%$ \\
\hline
\end{tabular}

Table 14: Clinical presentation in malignant tumors in various studies.

\begin{tabular}{|llllllll|}
\hline Study & Pain & $\begin{array}{l}\text { Mass } \\
\text { abdomen }\end{array}$ & $\begin{array}{l}\text { Menstrual } \\
\text { abnormality }\end{array}$ & Dyspepsia & $\begin{array}{c}\text { Ascites } \\
\text { aboss of }\end{array}$ & $\begin{array}{l}\text { Misc. } \\
\text { weight }\end{array}$ & $\begin{array}{l}\text { Mis. } \\
\text { Manoja et a }\end{array}$ \\
\hline Shanthi $\mathrm{V} \mathrm{et} \mathrm{al}^{7}$ & $25 \%$ & $25 \%$ & $16.7 \%$ & - & $8.3 \%$ & $16.7 \%$ & $8.3 \%$ \\
\hline Present study $(\mathrm{N}=162)$ & $30.77 \%$ & $23.08 \%$ & $23.08 \%$ & - & $7.69 \%$ & $15.38 \%$ & - \\
\hline
\end{tabular}

Pain in abdomen was the most common symptom in benign tumors $52 \%$ in present study while Manoja et al and Shanthi $\mathrm{V}$, reported mass abdomen to be the commonest symptom in benign tumors as shown in Table $13.1,7$

Among malignant ovarian tumors, dyspepsia 59.2\% was the most common symptom followed by mass abdomen $56.2 \%$ and pain $35.2 \%$. There were multiple symptoms present in many patients. Manoja et al and Shanthi $\mathrm{V}$ et al reported pain and mass in abdomen to be the commonest presenting symptom in their study in malignant tumors of ovary as shown in Table 14. ${ }^{1,7}$

\section{Laterality}

In present study benign tumors were unilateral in $68.36 \%$ cases while malignant tumors were unilateral in only $5.1 \%$ cases similar to the findings reported by Swamy $\mathrm{G}$ et al, where benign tumors were unilateral in $71 \%$ but $50 \%$ of malignant tumors also were unilateral. ${ }^{10}$ These findings were different from the findings of Manoja $\mathrm{V}$ et al, who reported unilateral benign tumors in $92.6 \%$ and $75 \%$ of malignant tumors also were unilateral as shown in Table 15 . $^{1}$

Table 15: Laterality of ovarian tumors.

\begin{tabular}{|c|c|c|c|c|}
\hline Study & Benign & & Malignant & \\
\hline & Unilateral & Bilateral & Unilateral & Bilateral \\
\hline Swamy G et al ${ }^{10}$ & $71 \%$ & $29 \%$ & $50 \%$ & $50 \%$ \\
\hline Manoja $\mathrm{V}$ et $\mathrm{al}^{1}$ & $92.6 \%$ & $7.4 \%$ & $75 \%$ & $25 \%$ \\
\hline Present study & $68.36 \%$ & $31.64 \%$ & $5.1 \%$ & $94.9 \%$ \\
\hline
\end{tabular}

Table 16: Histopathological pattern in various studies.

\begin{tabular}{|c|c|c|c|c|c|}
\hline Study & Surface epithelial & Sex cord stromal & Germ cell & Metastatic & Undifferentiated/others \\
\hline Yogambal et $\mathrm{al}^{2}$ & $71.64 \%$ & - & - & $0.75 \%$ & - \\
\hline Manoja et $\mathrm{al}^{1}$ & $84.2 \%$ & $4.2 \%$ & $10 \%$ & $0.8 \%$ & $0.8 \%$ \\
\hline Shanthi $\mathrm{V}$ et $\mathrm{al}^{7}$ & $84.62 \%$ & $3.85 \%$ & $10.9 \%$ & $0.64 \%$ & - \\
\hline Ameena et $\mathrm{al}^{8}$ & $52.76 \%$ & $3.15 \%$ & $43.31 \%$ & $0.78 \%$ & - \\
\hline Amod $\mathrm{S}$ et $\mathrm{al}^{9}$ & $84.8 \%$ & $6.1 \%$ & $9.1 \%$ & - & - \\
\hline Swamy G et al ${ }^{10}$ & $61.6 \%$ & $11.7 \%$ & $21.7 \%$ & $5 \%$ & - \\
\hline Sumaira $Y$ et al $^{12}$ & $76.5 \%$ & - & - & - & - \\
\hline Sarangan $\mathrm{A}$ et $\mathrm{al}^{11}$ & $81 \%$ & $4 \%$ & $15 \%$ & $0 \%$ & $0 \%$ \\
\hline Present study & $80.45 \%$ & $11.17 \%$ & $7.26 \%$ & $0.56 \%$ & $0.56 \%$ \\
\hline
\end{tabular}

The exact nature of tumor cannot be confirmed by just clinical examination. Tumor markers and imaging techniques are supportive measures in guiding the nature of tumor. The microscopic appearance is important and 
further management is guided by histopathological pattern. ${ }^{1,2}$ Grading of tumor is also important in further management. ${ }^{12}$

Among histopathological types the commonest tumor encountered in our study was epithelial tumor $(80.45 \%)$ followed by sex cord stromal tumors $(11.17 \%$ and germ cell tumors $(7.76 \%)$. All the studies have shown preponderance of surface epithelial tumors ranging from 61.6 to $82.8 \%$ followed by germ cell tumors. In our study sex cord stromal tumors were more than germ cell tumors. In study by Ameena et al, germ cell tumors were comparatively more constituting $43.3 \% .^{8}$ The authors contributed it to geographical variation. (Lahore) Table 16 shows the histopathological pattern of tumors in various studies.

Table 17: Proportion of tumors according to WHO classification.

\begin{tabular}{|c|c|c|c|c|c|c|c|}
\hline Histology & Manoja V & Shanthi V & Ameena & Amod S & Swamy G & Sumaira Y & Present study \\
\hline Surface epithelial & $80 \pm 4.2 \%$ & - & $26 \pm 26.76 \%$ & - & $47.4 \pm 14 \%$ & - & $39.4 \pm 41 \%$ \\
\hline Serous & $62.5 \pm 3.2$ & $64.75 \pm 1.92 \%$ & $15.7 \pm 11 \%$ & $54.5 \pm 18.2 \%$ & $40.8 \pm 6.6 \%$ & $24.5 \%$ & $48.47 \%$ \\
\hline Mucinous & $17.5 \pm 1$ & $16.67 \pm 1.3 \%$ & $10.3 \pm 7 \%$ & $6.1 \%$ & $6.6 \pm 4.9 \%$ & - & $19.38 \%$ \\
\hline Endometroid tumor & - & - & $0 \pm 5.51 \%$ & $6.1 \%$ & - & $28.5 \%$ & $01.53 \%$ \\
\hline Clear cell & - & - & $1.57 \%$ & - & - & - & - \\
\hline $\begin{array}{l}\text { Brenner tumor } \\
\text { (transitional cell) }\end{array}$ & - & - & $0 \pm 1.57 \%$ & - & - & - & $01.53 \%$ \\
\hline Mixed & - & - & - & - & $2.5 \%$ & - & - \\
\hline $\begin{array}{l}\text { Sex cord stromal } \\
\text { tumor }\end{array}$ & $0.8 \pm 3.4 \%$ & $1.28 \pm 2.56 \%$ & $0.8 \pm 2.35 \%$ & $6.1 \%$ & - & - & $9.5 \pm 1.6 \%$ \\
\hline Steroid cell & - & - & $0.8 \%$ & - & - & - & - \\
\hline Sertoli leydig cell & $0.8 \%$ & $0.64 \%$ & - & - & - & - & - \\
\hline \multicolumn{8}{|l|}{ Adeno-fibroma } \\
\hline Fibroma thecoma & $0.8 \%$ & $1.28 \%$ & - & $6.1 \%$ & - & - & - \\
\hline Granulosa cell & $1.7 \%$ & $1.28 \%$ & $2.27 \%$ & - & - & $28.5 \%$ & - \\
\hline $\begin{array}{l}\text { Mixed mullerian } \\
\text { tumor }\end{array}$ & 0.8 & 0.64 & - & - & - & - & - \\
\hline Germ cell tumor & $9.2 \pm 0.8$ & $8.97 \pm 1.92$ & $37.8 \pm 5.5$ & $9.1 \%$ & - & - & $5.86 \pm 1.4 \%$ \\
\hline $\begin{array}{l}\text { Mature cystic } \\
\text { teratoma }\end{array}$ & $9.2 \%$ & $8.97 \%$ & $37.8 \%$ & $9.1 \%$ & - & $18 \%$ & - \\
\hline Dysgerminoma & - & $1.28 \%$ & $1.59 \%$ & - & - & - & - \\
\hline Immature teratoma & $0.8 \%$ & $0.64 \%$ & $1.59 \%$ & - & - & - & - \\
\hline Yolk sac tumor & - & - & $2.27 \%$ & - & - & - & - \\
\hline $\begin{array}{l}\text { Secondary } \\
\text { (Krucken berg) }\end{array}$ & $0.8 \%$ & $0.64 \%$ & $0.8 \%$ & - & - & - & $0.56 \%$ \\
\hline Undifferentiated & $0.8 \%$ & - & - & - & - & - & $0.56 \%$ \\
\hline
\end{tabular}

Table 18: Comparison of distribution of ovarian tumors in different age groups in various studies.

\begin{tabular}{|llllll|}
\hline Age & Manoja V & Shanthi $\mathbf{V}^{\mathbf{7}}$ & Ameena & Sarangan A & Present study \\
\hline$<20$ years & $11.7 \%$ & $10.26 \%$ & $12.21 \%$ & $2 \%$ & $1.1 \%$ \\
\hline 21-30 years & $25 \%$ & $25.64 \%$ & $30.19 \%$ & $24 \%$ & $7.8 \%$ \\
\hline 31-40 years & $29.2 \%$ & $28.85 \%$ & $22.64 \%$ & $29 \%$ & $24.8 \%$ \\
\hline 41-50 years & $18.3 \%$ & $18.59 \%$ & $18.40 \%$ & $27 \%$ & $44.4 \%$ \\
\hline 51-60 years & $9.2 \%$ & $9.62 \%$ & $10.38 \%$ & $13 \%$ & $20.8 \%$ \\
\hline$>60$ years & $6.6 \%$ & $7.0 \%$ & $5.18 \%$ & $5 \%$ & $1.1 \%$ \\
\hline
\end{tabular}

When the tumors were categorised according to WHO subtypes, serous epithelial tumors were more common in both benign and malignant category followed by mucinous tumors as shown in Table 17.
Age

The mean age in benign epithelial tumors in present study was $42.06 \pm 5.8$ years while malignant tumors presented at mean age of $52.97 \pm 6.27$ years. Deeba et al reported mean 
age as $40.6 \pm 12.5$ years in ovarian cancer cases while in study by Ameena A et al mean age at presentation of ovarian tumors was 35.6 years. Shanthi V, reported preponderance of benign tumors in 21-50 years similar to present study and malignant tumors were more common after 40 years. $^{7}$ The age comparison of ovarian tumors in various studies is as shown in Table 18. All studies show similar pattern of occurrence of ovarian tumors that is preponderance in 21-50 years of age.

\section{CONCLUSION}

It is concluded from this study that the tumors originating from surface epithelium are the commonest variant. Germ cell tumours and sex cord stromal tumors were next to epithelial ovarian tumours. Majority of them were benign. Amongst malignant ovarian tumours late reporting is common and patients usually present in advanced stages of the disease. Commonest clinical presentation was mass abdomen and pain abdomen. Though the imaging technique and clinical examination help in detecting ovarian tumors, histopathological examination is the gold standard to determine the type of the ovarian tumor and its histogenesis which affects the treatment and prognosis of the tumor.

\section{Funding: No funding sources}

Conflict of interest: None declared

Ethical approval: The study was approved by the Institutional Ethics Committee of Goa Medial College, Goa, India

\section{REFERENCES}

1. Manoja V, Pramood M, Jyothi V, Chandrashekar KPA. Clinicopathological study of ovarian tumors: a 2-year study. Int J Sci Stud. 2017;5(3):300-5.

2. Yogambal M, Arunalatha P, Chandramouleeswari K, Palaniappan V. Ovarian tumours- incidence and distribution in a tertiary referral center in south India. IOSR J Dent Med Sci. 2014;13(2):74-80.
3. Ferlay J, Soerjomataram I, Ervik M, Dikshit R, Eser S, Mathers C, et al. GLOBOCAN 2012 v1.0, Cancer Incidence and Mortality Worldwide: IARC CancerBase No. 11. Lyon, France: International Agency for Research on Cancer (IARC); 2013.

4. Reid BM, Permuth JB, Sellers TA. Epidemiology of ovarian cancer: a review. Cancer Biol Med. 2017;14(1):9-32.

5. Sharma M, Soni A, Kaul R. Histopathological pattern of ovarian neoplasms in Sub-Himalayan belt of rural India: a four-year study from a tertiary care teaching hospital. Int $\mathbf{J}$ Reprod Contracept Obstet Gynecol. 2017;6:5448-52.

6. Deeba F, Alam ABMM, Banu J. Clinlcopathological study of ovarian cancer: a multi centered study. J Shaheed Suhrawardy Med Coll. 2013;5(1):3-6.

7. Shanthi V. Clinico pathological study of ovarian tumors- a retrospective and prospective 5 years study. JMSCR. 2015;4(6):10880-5.

8. Ashraf A, Shaikh AS, Ishfaq A. The relative frequency and histopathological pattern of ovarian masses. Biomedica. 2012;28:98-102.

9. Sawant A, Mahajan S. Histopathological study of ovarian lesions at a tertiary health care institute. MVP J Med Sci. 2017;4(1):26-9.

10. Swamy GG, Satyanarayana N. Clinicopathological analysis of ovarian tumors: a study on five years Samples. Nepal Med Coll J. 2010;12(4):221-3.

11. Sarangan. "Clinicopathological and histological features of ovarian tumour- a study." IOSR J Dent Med Sci. 2017;16(9):56-60.

12. Yasmin S, Yasmin A, Asif M. Clinicohistological pattern of ovarian tumours in Peshawar region. J Ayub Med Coll Abbottabad. 2008;20(4);11-3.

Cite this article as: Jindal M, Jindal D,

Sahasrabhojane M, Naik V. Clinico-pathological study of ovarian tumors in Goa medical college: a tertiary care centre in Goa, India. Int J Reprod Contracept Obstet Gynecol 2019;8:4071-8. 